\title{
Responsibility in life cycle assessment practice
}

\author{
Martin Baitz ${ }^{1}$ \\ Received: 6 November 2018 / Accepted: 8 November 2018 / Published online: 3 December 2018 \\ (C) Springer-Verlag GmbH Germany, part of Springer Nature 2018
}

People are strange when it comes to aging - everybody wants to mature, but nobody wants to grow old. Certain LCA practitioners seem to embody similar ways of thinking when it comes to taking responsibility for methods, software, data, and the communication of results - everybody wants responsibly generated LCA facts, but few are willing to do what is necessary to take responsibility for them.

In my 24 years working in LCA, I have witnessed many incidents, starting in research and academia, moving through a start-up company and today as part of a small- and mediumsized global corporation. In most cases, incidents arose when one or more of the parties involved failed to take responsibility for the LCA setup, data procurement, analysis, or the resulting communication.

And what if this were to play out in a trial? As you will later discover in this article, this actually happened.

I wish to exchange experiences with peers, colleagues, and competitors on the topic of "Responsibility in LCA." I have already spoken with accomplished professionals in the LCA community from the Netherlands, Switzerland, and the USA about this topic. There seems to be a fair amount of consensus about the need for and benefit of diving deeper into best practices around responsibility in LCA as a means of protecting LCA as an approach from unjustified criticism and so as to safeguard users from flawed LCA results and conclusions.

The following paragraphs touch on different types of responsibility and aim to encourage and provoke thought about this very topic.

The LCA results may not be what the client expected Contractual partners are responsible for sticking to the

Responsible editor: Mary Ann Curran

Martin Baitz

martin.baitz@thinkstep.com

1 thinkstep AG, Hauptstr. 111-113, 70771 Leinfelden-Echterdingen, Germany technical specifications and deliverables defined at the beginning of a project and documented in its goals and scope. This seems straightforward, but in LCA often the resulting conclusions are unforeseeable at first sight and the desired outcome may drift in a direction that may not support the initially desired intention, message or follow-up actions. Twenty years back, a single association in a multi-client project refused to accept the verified results of an LCA project. However, the carefully defined goal and scope, including the technical description of the deliverables, was ultimately convincing enough to remind them that even unexpected LCA results can be used responsibly to improve and derive benefits. So everybody should take unexpected LCA results as a chance to improve, as you would accept an unexpected diagnosis from your doctor as the first step toward a cure.

Software, data, and methods should be fully cited One of the most important responsibilities of any user is to carefully reveal the basis of his or her work. There are still LCA users out there who cite the software they used as "GaBi" or "Simapro." Any data used is often just cited as being from the "thinkstep database" or from the "ecoinvent database" and the impact method used is simply called "CML" or "EPS." Such generic citing is irresponsible. Software has a version number, databases have release years, and most methods have either a version number or a release year.

Ensure background data support the goal and scope Users should read the dataset documentation to check if the chosen background data fit the purpose of the goal and scope. Just looking up the dataset name, such as "steel," "aluminum," "PVC," or "paper," is insufficient and does not reflect the needed precision if you want the results to be interpreted reliably.

Dig deep into the results When looking at practical relevance, LCA practitioners should move beyond the simple results and dig deeper into the technical or practical implications of those results. The simple communication that "less emissions are 
potentially preferable" is just not good enough. LCA practitioners have to be brave enough to link their results back to the technical implications and chances for improvement, explaining why and how far material options, design options, and process options are linked to the LCA results.

Keep it as simple as possible If we want LCA results to be taken seriously, we also need to avoid modelling overcomplicated or theoretically extended systems - we need to stop pretending that LCA can only be managed by so-called experts. We need to avoid communicating inadequate results based on pure model theory and communicate results based on engineering reality. LCA practitioners need to focus their models on the core aspects, so that LCA results can be turned into responsible technical testimonies. Here, I might pause to quote Einstein, "You do not really understand something unless you can explain it to your grandmother."

Review and compare responsibly This is particularly important when people benchmark, compare, or test different existing LCA technology - like software and databases - or when they elaborate on various published studies that have different conclusions. To protect the reviewed technology or study from unjustified criticism and to prevent the reviewing party from publishing inaccurate facts (or even protect them from lawsuits), it is critical for the reviewer to thoroughly check the comparability, the consistency, and the resulting review conclusions. Therefore, before publishing such "critical" reviews or comparisons, it is highly recommendable to inform the original source or developer about the findings and ask for comments.

Concerning the communication of results, I remember a surprising call from an industry company. They were astonished to read my name as a critical reviewer under a rather blunt statement like, "This product is carbon neutral." I was also astonished. A study that conformed perfectly to ISO standards and had ten suitable result scenarios with careful interpretation was sliced and diced into pieces by a marketing department. Only the best (and least likely) scenario was communicated as the one and only result. Responsible marketing of LCA results should avoid cherry picking and perhaps people should consider including LCA result generators in their marketing to ensure the initial (reviewed) interpretations. By the way, an ISO conforming review statement-like the one issued for the industry company in the above example-must always refer to the complete study report and never to isolated aspects of a study.

The most dramatic case concerning responsibility was a two-year lawsuit about the results of a study undertaken by my company years back. Under the defined goal and scope, one of the studied options was less preferable than the other two options. The issue started with a representative from the "winning option" prominently communicating that choice as being generally favorable, failing to mention the specific goal and scope of that option. It took only three days for an injunction to be issued and for a lawsuit to start against the authors of the study. The plaintive claimed that there were numerous mistakes and false data points. As far as we know, it was the first LCA study examined at a trial. It proved quite interesting to be forced to inform and educate judges and lawyers concerning LCA and to prove our points. The case even went from a lower court to the highest German court, the Federal Administrative Court in Leipzig. Suddenly, you realize how many topics people need to take responsibility for to make an LCA airtight: Starting with a clear goal and scope and expanding into suitable data sources, the actual data, appropriate benchmarks as points of evidence, justifiable assumptions, realistic scenarios, reliable interpretations, and a solid panel review with suitable professionals who act as "representatives" of the different options.

After two years of accusations against the authors of the study, the lawsuit was dropped. All the claimed mistakes and supposedly false data points were disproved, except for a single recycling rate that had to be corrected from 77 to $80 \%$. The trial was a victory for my company and especially the authors of the LCA report. However, the person who caused the issues with generalized result communication did learn a costly lesson-how to communicate responsibly next time.

So again, I want to use this as a starting point for reaching out and initiating further exchange between peers, colleagues, and competitors on the topic of responsibility in LCA. I am sure there are many more worthwhile examples we can discuss and act on, and I am convinced it will help the LCA community as a whole and increase the likelihood that more people will take responsibility in LCA. Dare to do it.

Publisher's Note Springer Nature remains neutral with regard to jurisdictional claims in published maps and institutional affiliations. 